\title{
La minute du superviseur
}

\section{Jean JOUQUAN}

Bureau de pédagogie médicale, Faculté de médecine, Université de Bretagne occidentale, Brest, France

\begin{abstract}
Définition
Procédure de supervision d'un étudiant (interne, externe), visant à concilier les deux objectifs de la supervision clinique en contexte de stage : a) assurer l'efficacité et la sécurité des tâches de soins dispensés au patient (finalité médicale de la supervision); b) faciliter l'apprentissage de l'étudiant à partir de cette tâche professionnelle (finalité pédagogique de la supervision).

\section{Problématique}

Deux modalités de supervision sont couramment employées dans le cadre de la formation en contexte de stage. La supervision est dite «directe» lorsque la rétro-action fournie à l'étudiant succède à une phase d'observation directe de l'étudiant dans sa tâche clinique. La supervision est dite «indirecte » lorsque l'interaction avec l'étudiant est développée à partir de la présentation que fait ce dernier de la situation clinique, sans que l'enseignant clinicien l'ait personnellement observé. Plusieurs études indiquent qu'une séquence de supervision indirecte d'un étudiant à propos d'un patient dure approximativement dix minutes. Sur ce temps, environ six minutes sont consacrées à la présentation de la situation clinique par l'étudiant et trois à des questions du superviseur visant à clarifier les données ; ainsi, en moyenne, la discussion visant à exploiter pédagogiquement la situation pour faciliter les apprentissages de l'étudiant à partir de sa tâche clinique se limite à une seule minute. Le format dit de « la minute du superviseur» (the One Minute Preceptor) a été codifié pour optimiser cette «minute pédagogique », au décours immédiat de la présentation du cas par l'étudiant.

\begin{tabular}{|l|}
\hline \multicolumn{1}{|c|}{ Description } \\
\hline Format princeps \\
Le format princeps de la minute du superviseur a été \\
formalisé en 1992 par Neher et al. ${ }^{[1]}$, au Département \\
de médecine de famille de l'Université de Washington, \\
sous la forme d'une séquence de cinq étapes : 1) prise \\
\hline
\end{tabular}

de position ; 2) argumentation ; 3) énoncé d'une règle générale; 4) validation des points positifs; 5) correction des erreurs. Ces étapes, complétées par une conclusion, fournissent un cadre opératoire sous-tendu par des principes pédagogiques visant à favoriser des apprentissages transférables. Cette séquence est mise en œuvre après que l'étudiant ait rapporté la situation. Le tableau I indique les objectifs cognitifs de chaque étape, fournit quelques exemples d'interventions types et indique quelques écueils à éviter.

\section{Variantes}

Les étapes 3 à 5 peuvent éventuellement être interverties. L'étape 2 (argumentation) peut être exploitée par le superviseur pour effectuer un travail de décontextualisation et de recontextualisation, qui permet de favoriser la construction de connaissances conditionnelles grâce aux stratégies de discrimination (en quoi deux situations apparemment semblables se distinguent-elles en fait?) et de généralisation (en quoi deux situations apparemment différentes sont-elles en fait semblables ?) et de préparer le transfert des apprentissages.

\section{Modalités opérationnelles}

Plusieurs exemples de mise en œuvre de la minute du superviseur sont disponibles dans la littérature francophone ${ }^{[2,3]}$ ou internationale ${ }^{[4]}$. Des séquences vidéo de démonstration sont également disponibles ${ }^{[5]}$.

\section{Conclusion}

Le format de «la minute du superviseur» fournit un cadre opératoire de supervision clinique commode à mettre en œuvre. Il est cohérent avec les principes pédagogiques visant à favoriser des apprentissages transférables. Il ne se substitue pas à d'autres modalités plus élaborées d'intervention pédagogique en contexte de stage. À condition de ne pas être utilisé comme une recette technique, il peut apporter des repères utiles aux enseignants cliniciens lorsqu'ils doivent, dans le cadre de contraintes de temps importantes, concilier leur deux rôles professionnels de clinicien et de formateur ${ }^{[6]}$. 


\section{Références}

1. Neher JO, Gordon KC, Meyer B, Stevens N. A five-step "microskills" model of clinical teaching. J Am Board Fam Pract 1992;5:419-24.

2. Audetat M-C, Laurin S. Clinicien et superviseur... Même combat ! Le Médecin du Québec 2010;45:53-7.

3. Belleflamme M, Boulouffe C, Gérard V, de Cannière L, Vanpee D. Comment développer le raisonnement clinique des étudiants en stage à l'hôpital quand on dispose de peu de temps ? "The One-Minute Preceptor". Louvain Médical 2009;128:165-9.

4. Sarkin R. The One-Minute preceptor. Microskills of clinical teaching. [On-line]. Disponible sur : http://www . im . org/ Resources/FacultyDev/GIMFDP/Documents/Strategies\%20Tampa\%20Sarkin.htm.

5. Promoting effective clinical teaching. One Minute Preceptor in action. [On-Line] Disponible sur : http://www . practicalprof.ab.ca/teaching_nuts_bolts/one_minute_preceptor.html\#.

6. Aagaard E, Teherani A, Irby D. Effectivness of the One-Minute Preceptor Model for diagnosing the patient and the learner: proof of concept. Acad Med 2004;79:42-9.

Tableau I. Les cinq étapes du format princeps de «la minute du superviseur ».

\begin{tabular}{|c|c|c|c|}
\hline Étapes de la supervision & Objectifs de l'étape & Exemples d'intervention & Écueils à éviter \\
\hline $\begin{array}{l}\text { 1. Inviter l'étudiant à prendre po- } \\
\text { sition (en formulant une hypothèse } \\
\text { diagnostique, en proposant une dé- } \\
\text { marche d'investigation ou une déci- } \\
\text { sion thérapeutique) }\end{array}$ & $\begin{array}{l}\text { Solliciter les connaissances antérieures } \\
\text { de l'étudiant et notamment ses connais- } \\
\text { sances d'action (conditionnelles et pro- } \\
\text { cédurales) }\end{array}$ & $\begin{array}{l}\text { Quelle est ta compréhension du pro- } \\
\text { blème de santé de ce patient? } \\
\text { Que proposes-tu de faire? } \\
\text { De quel examen complémentaire } \\
\text { penses-tu que nous ayons besoin? }\end{array}$ & $\begin{array}{l}\text { Fournir d'emblée à l'étudiant la solu- } \\
\text { tion du problème, sans solliciter préa- } \\
\text { lablement sa propre représentation et } \\
\text { ses propres hypothèses }\end{array}$ \\
\hline $\begin{array}{l}\text { 2. Demander à l'étudiant d'argumen- } \\
\text { ter sa position }\end{array}$ & $\begin{array}{l}\text { Rendre explicite le processus de raison- } \\
\text { nement clinique développé par l'étu- } \\
\text { diant }\end{array}$ & $\begin{array}{l}\text { Qu'est-ce qui te permet d'arriver à } \\
\text { cette conclusion? } \\
\text { Pourrait-il y avoir une autre explica- } \\
\text { tion? }\end{array}$ & $\begin{array}{l}\text { Faire réciter des connaissances décla- } \\
\text { ratives de manière décontextualisée }\end{array}$ \\
\hline $\begin{array}{l}\text { 3. Énoncer une règle } \\
\text { générale }\end{array}$ & $\begin{array}{l}\text { Décontextualiser les } \\
\text { connaissances mobilisées }\end{array}$ & $\begin{array}{l}\text { Dans un contexte de faible présomp- } \\
\text { tion clinique, ce diagnostic peut être } \\
\text { écarté lorsque tel signe est absent, } \\
\text { compte tenu de son excellente valeur } \\
\text { prédictive négative }\end{array}$ & $\begin{array}{l}\text { Délivrer des messages anecdotiques } \\
\text { ou fondés sur des habitudes idiosyn- } \\
\text { crasiques }\end{array}$ \\
\hline $\begin{array}{l}\text { 4. Valider les éléments pertinents de } \\
\text { la prise en charge proposée par l'étu- } \\
\text { diant }\end{array}$ & Renforcer les apprentissages viables & $\begin{array}{l}\text { Ton inventaire des facteurs de gravité } \\
\text { est tout à fait pertinent et complet; tu } \\
\text { n'as rien oublié }\end{array}$ & $\begin{array}{l}\text { Fournir une rétro-action générale, } \\
\text { non spécifique («OK, c’est bien !») }\end{array}$ \\
\hline $\begin{array}{l}\text { 5. Identifier les erreurs et suggérer } \\
\text { des solutions alternatives }\end{array}$ & $\begin{array}{l}\text { Déconstruire les conceptions erronées } \\
\text { Recontextualiser les connaissances mo- } \\
\text { bilisées }\end{array}$ & $\begin{array}{l}\text { Ne penses-tu pas que tu as pris un } \\
\text { risque excessif en ne demandant pas tel } \\
\text { examen complémentaire en urgence? } \\
\text { Comment pourrais-tu procéder une } \\
\text { autre fois dans un cas semblable? }\end{array}$ & $\begin{array}{l}\text { Se limiter à un jugement négatif et } \\
\text { dévalorisant sans rendre explicite les } \\
\text { représentations qui ont sous tendues } \\
\text { l'action (« Comment, tu as fait cela! } \\
\text { C'est nul!») }\end{array}$ \\
\hline Conclusion & $\begin{array}{l}\text { Planifier les étapes ultérieures } \\
\text { Identifier les rôles respectifs } \\
\text { Identifier les indicateurs } \\
\text { de suivi ou d'alerte }\end{array}$ & $\begin{array}{l}\text { Recontacte-moi dès que tu auras le ré- } \\
\text { sultat de tel examen et après avoir ap- } \\
\text { pelé le médecin traitant. On réévaluera } \\
\text { nos hypothèses. } \\
\text { Précise bien les consignes de sur- } \\
\text { veillance pour l'interne de garde cette } \\
\text { nuit }\end{array}$ & \\
\hline
\end{tabular}

Correspondance et offprints : Jean Jouquan, Bureau de pédagogie médicale, Faculté de médecine, Université de Bretagne occidentale, Brest, France.

Mailto : jean.jouquan@chu-brest.fr 